\title{
Structure Analyses and Flow Rate Model of Peristaltic Pump of Cement Foaming Machine
}

\author{
Xiue Wu, Qingzhu Cui \\ ${ }^{1}$ University of Science and Technology Liaoning 114051, China \\ 2 Liaoning Shihua University 113001 , China \\ wuxiue2008@163.com, melody5481@163.com
}

Key Words: foam cement, peristaltic pump, flow rate

\begin{abstract}
With the constant development in the fields of Greenness, Environmental Protection and Energy Conservation all over the world, the improvement of foam cement technology is to bring about a revolution in the architectural material industry. The main component of the cement foaming machine is the delivery pump system, in which the peristaltic pump is the most important part. In this thesis, on the bases of study of peristaltic pump in imported machines, mathematical models are made and an approximate calculation of flow rate of the peristaltic pump is given through exhaustive analyses of the structure and the changes in the cross section before and after compression rolling. This research will also make it possible to analyze the pressure in the exit of the pump and deduce the power needed by the driven peristaltic pump according to the factors like the calculation of on-way pressure loss with the consideration of working altitude. This research is expected to provide a theoretic principle for the development of cement foaming technology.
\end{abstract}

\section{Introduction}

Cement foaming technology, which originated overseas and was introduced into China more than 10 years ago, is still in its initial stage of development. While, with numerous close interspaces, foam cement enjoys outstanding physical and mechanical advantages of light weight, heat preservation, thermal insulation, sound insulation and flame resistance etc. Therefore, we have reason to believe that with the constant development in the fields of Greenness, Environmental Protection and Energy Conservation all over the world, the improvement of foam cement technology will bring about a revolution in the architectural material industry. Cement foaming machine is the equipment operated to foam, deliver, and concrete. The delivery pump system serves as the main component of the cement foaming machine, because foam cement grout is sticky and contains grain, peristaltic pumps are needed to pump and pour.

Traits of peristaltic pumps. Peristaltic pumps fall into the category of positive displacement pump. Their unique non-seal structure which prevents it from leaking and other strong points, such as strong self-suction capacity, high lift coefficient and simple structure make them suitable for delivering high viscous, caustic and high abrasive materials. Their flexibility of turning contributes to their functions of anti-block and self-clearing.

Structure and working principle of peristaltic pumps. Peristaltic pump (Fig. 1) is mainly composed of pump shell, tube, compression roll, delivery roll and back-up roll. Its working principle is as follows: the electromotor drives the rotor, on which the three compression rolls press the material along the axis of the tube. Then because of the elasticity and the force of the delivery roll and back-up roll, the tube come back to its original shape and this state of vacuum draw the liquid in, and at the same time, the fluid in the is pressed out of the tube.

The calculations of flow rate in peristaltic pumps. The structure design and calculations of flow rate with a sound theoretical basis is yet to come. The calculation in this paper is modeled and given in reference to the foam device imported from Korea. 


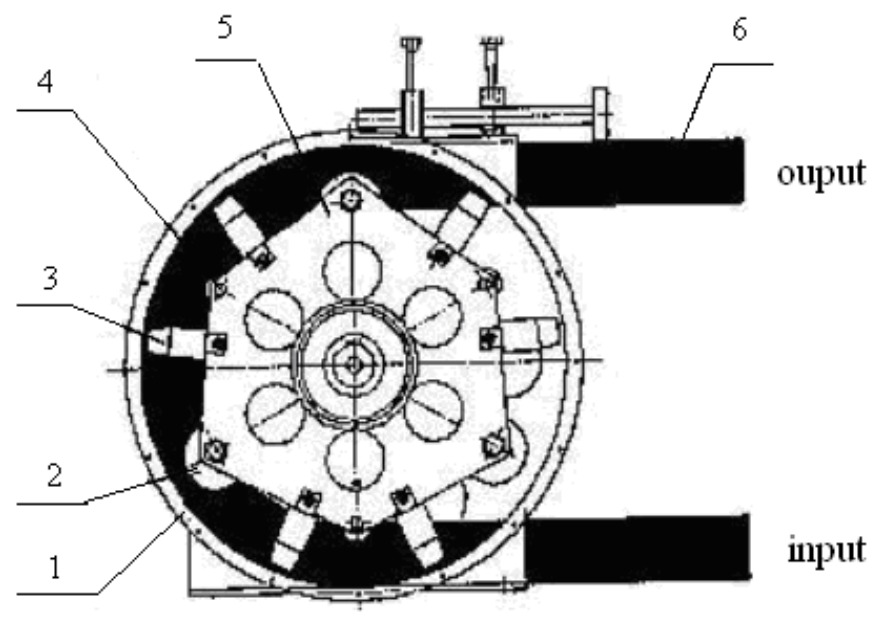

1- pump shell 2- compression roll 3- delivery roll 4-back-up roll 5-rotor 6-tube

Fig. 1. Structure of peristaltic pump

The simplified shapes of the cross section of peristaltic pumps before and after compress are shown in Fig. 2. The axial curvature of compression roll is so neglectable that it can be seen as a straight line. The above curve can still be seen as an arc, only the curvature diminished.

Equation of the curve in the tube:

$$
x^{2}+(y+a)^{2}=(r+a)^{2} \text {. }
$$

Parameter equation:

$$
\begin{aligned}
& x=(r+a) \cos \varphi \\
& y=(r+a) \sin \varphi-a
\end{aligned}
$$

$r:$ the inner radius

$a:$ the down moved distance of the transformed arc center

Now $\varphi_{0}$ and $a$ will be determined:

The perimeter of the tube remain the same before and after change, so

$$
\begin{aligned}
& (r+a) \cos \varphi_{0}+(r+a)\left(\frac{\pi}{2}-\varphi_{0}\right)=r \pi \\
& \because \quad \sin \varphi_{0}=\frac{r+a-h}{r+a}
\end{aligned}
$$

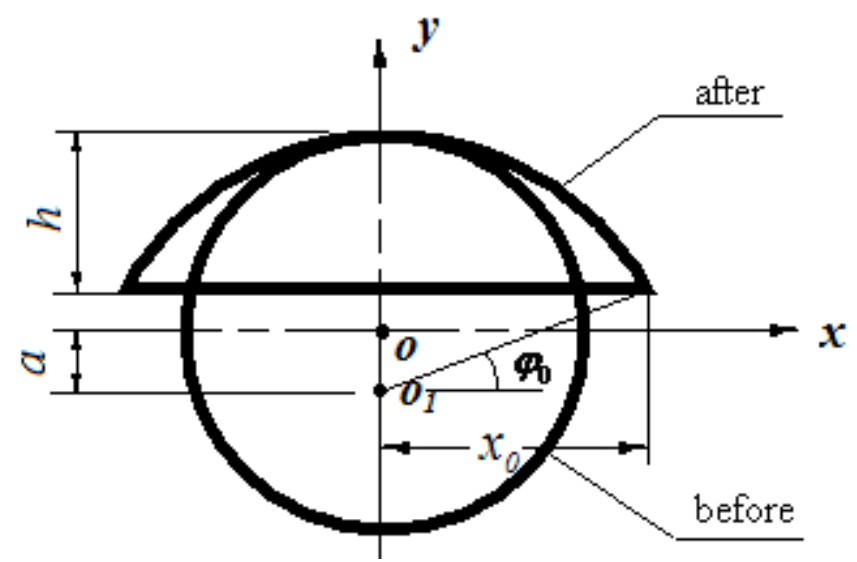

Fig. 2. Cross section of peristaltic pumps before and after compress 


$$
\therefore \quad a=\frac{h}{1-\sin \varphi_{0}}-r
$$

where, $h$-midpoint height of inner circle after change, this numerical value is determined by structural size.

Put (3) into (2), can be developed to

$$
h\left(\cos \varphi_{0}+\frac{\pi}{2}-\varphi_{0}\right)-\pi r\left(1-\sin \varphi_{0}\right)=0
$$

As the actual $\varphi_{0}$ is very small, develop $\sin \varphi, \cos \varphi$ to Maclaurin progression, and make rough equations

$$
\sin \phi=\phi-\frac{\phi^{3}}{3 !}, \quad \cos \phi=1-\frac{\phi^{2}}{2 !} .
$$

Put (5) into (4), can be developed to

$$
\frac{\pi r}{6} \varphi_{0}^{3}+\frac{h}{2} \varphi_{0}^{2}+(h-\pi r) \varphi_{0}+\left(\pi r-h-\frac{\pi}{2} h\right)=0
$$

When $r$ and $h$ are given, with method Newton-Raphson in Bibliography [5], the result of parameter $\varphi_{0}$ is available with (6), and the initial value $a$ can be decided by (3).

Cross section area of inner circle after change

$$
A=2 \int_{0}^{x_{0}}[y-(r-a-h)] d x
$$

Where, $y=(r+a) \sin \varphi-a$

$$
x=(r+a) \cos \varphi, \quad d x=-(r+a) \sin \varphi d \varphi,
$$

When $x=0, \varphi=\frac{\pi}{2}$, and when $x=x_{0}, \varphi=\varphi_{0}$.

$$
\begin{aligned}
\therefore \quad A & =-2 \int_{\frac{\pi}{2}}^{\varphi_{0}}[(r+a) \sin \varphi d \varphi-r+h](r+a) \sin \varphi d \varphi \\
& =(r+a)\left[2(h-r) \cos \varphi_{0}-\frac{r+a}{2}\left(2 \varphi_{0}-\sin 2 \varphi_{0}-\pi\right)\right]
\end{aligned}
$$

Cross section area of inner circle before and after change

$\Delta A=\pi r^{2}-A$

So, the flow rate of peristaltic pump

$Q=\Delta A \cdot \pi r \cdot n$

in which, $\mathrm{n}$-the number of compress roll.

The exist press ure of peristaltic pump

$P=P_{0}+\rho g h+\Delta P$.

where, $P_{0}$ - the atmosphere pressure of the nozzle 
$\rho$ - density of foam cement

$\mathrm{h}$-working height

$\Delta P$ - pressure loss

$\Delta P=\lambda \frac{\rho l v^{2}}{4 r}$

$\lambda=\frac{64}{\operatorname{Re}}=\frac{32 \mu}{\rho v r}$

$\therefore$ pressure at the exit $P=P_{0}+\rho g h+\frac{8 l v \mu}{r^{2}}$

where, 1 - length of the tube

$\mathrm{v}$ - average floating rate of

$\mu$-moving viscosity of cement fluid )

\section{Summary}

On the bases of the analyses of the structure of imported peristaltic pump, a calculation of exist pressure is made with neglectable error in practice. The function of peristaltic pump peristaltic pumps also extends to other fields such as chemical industry, medicine and pharmacology, metallurgy, petroleum. So, we can safely predict that this research will provide a theoretical basis for the development of peristaltic pumps, the improvement of foam cement and the design of related fields.

\section{References}

[1] P.L. Li. Main purpose and design calculation of the RGB tube pump,Machine.25 (1998) pp 29-30.

[2] W.X. Wang, Applications of Foamed Concrete in Housing Construction, Housing Materials \& Applications, 3(1998), pp 3-6.

[3] X.C. Zhou, Experimental Hose Pump and Its Experimental Design, Forestry Machinery \& Woodworking Equipment, 9 (2006), pp. 10-12.

[4] H.T. Zhu. Transmission of hydraulic and air pressure. Tsinghua University Press.2005.

[5] C,S. Lin, M. Mathematical Method, Science and education Press, 2001. 